\title{
Sex-Related Differences in the Risk Factor Profile and Medications of Patients With Atrial Fibrillation Recruited in J-TRACE
}

\author{
Hiroshi Inoue, MD ${ }^{1}$; Takashi Nozawa, MD ${ }^{1}$; Tadakazu Hirai, $\mathrm{MD}^{1}$; Shinya Goto, MD²; \\ Hideki Origasa, $\mathrm{PhD}^{3}$; Kazuyuki Shimada, $\mathrm{MD}^{4}$; Shinichiro Uchiyama, $\mathrm{MD}^{5}$; \\ Takayuki Hirabayashi, $\mathrm{MD}^{6}$; Yukihiro Koretsune, $\mathrm{MD}^{7}$; Shiro Ono, $\mathrm{MD}^{8}$; \\ Tooru Hasegawa, MD ${ }^{9}$; Yasuo Sasagawa, MD ${ }^{10}$; Yoshiaki Kaneko, MD $^{11}$; \\ Yasuo Ikeda, $\mathrm{MD}^{12}$ for the J-TRACE Investigators
}

\begin{abstract}
Background: Clinical characteristics, including risk factors for thromboembolism, and medications differ between men and women with atrial fibrillation (AF) in Western countries. Whether such a difference exists for Japanese patients with AF is unclear, so data from J-TRACE were used to investigate this issue.

Methods and Results: A total of 2,892 patients (2,028 men, 864 women; 70.3 years old) with AF were analyzed for the respective prevalences of risk factors and medications. CHADS2 score was calculated to determine thromboembolic risk level. Women were older $(\mathrm{P}<0.001)$, and more frequently had heart failure $(\mathrm{P}<0.001)$, and hypertension $(P=0.051)$ than men. The proportion of subjects aged 75 years or older was higher among women than among men $(P<0.001)$. CHADS2 score was therefore significantly higher in women than in men $(2.05 \pm 1.29$ vs $1.88 \pm 1.33, \mathrm{P}<0.001)$. Sex-related differences were not observed for the prevalence of diabetes mellitus, myocardial infarction or ischemic stroke, nor did warfarin usage differ between men and women.
\end{abstract}

Conclusions: Sex-related differences were observed in the risk factor profile and medications of Japanese patients with AF. CHADS2 score was higher in women than in men. (Circ J 2010; 74: 650-654)

Key Words: Atrial fibrillation; CHADS2 score; Clinical characteristics; Medications; Sex differences

A trial fibrillation $(\mathrm{AF})$ is a common cardiac arrhythmia seen in general practice as well as in the cardiology clinic. The prevalence of AF differs between men and women in Western countries, ${ }^{1-3}$ and also in Japan. ${ }^{4,5}$ Several studies have reported that there are sex-related differences in the clinical characteristics and medications of patients with $\mathrm{AF}^{6-10}$ A prospective, cohort study indicated that the effects of AF on the risk of stroke were greater in women than in men after adjustment for age and comorbidity. ${ }^{9}$ Other studies also showed that AF is associated with an increase in cardiovascular events, including mortality and stroke, especially in women.,11,12 Some risk stratification schemes consider women to be at high risk for ischemic stroke, ${ }^{13,14}$ while others do not. ${ }^{15,16}$ However, because the sex-related differences in risk factors for cardiovascular dis- eases and medications of Japanese patients with AF have yet to be clarified, registry data for a large, nation-wide, multicenter, cooperative study, J-TRACE (The Japan Thrombosis Registry for Atrial Fibrillation, Coronary or Cerebrovascular Events), ${ }^{17,18}$ were analyzed to address this issue in the present study.

\section{Methods}

The details of J-TRACE have been reported elsewhere. ${ }^{17,18}$ Briefly, J-TRACE has a steering committee of 5 members and 41 regional coordinators selected from 10 regions of Japan (Appendix 1). Recruitment of patients to investigate risk factor profiles and current status of medications for risk factors and for prevention of cardiovascular events in patients with

Received November 4, 2009; revised manuscript received December 18, 2009; accepted December 21, 2009; released online February 20, 2010 Time for primary review: 19 days

${ }^{1}$ Department of Internal Medicine, University of Toyama, Toyama, ${ }^{2}$ Department of Internal Medicine, Tokai University, Isehara, ${ }^{3}$ Department of Biostatistics, University of Toyama, Toyama, ${ }^{4}$ Department of Cardiology, Jichi Medical University, Shimotsuke, ${ }^{5}$ Department of Neurology, Tokyo Women's Medical University, Tokyo, ${ }^{6}$ Department of Cardiology, Sunagawa City Medical Center, Sunagawa, ${ }^{7}$ Institute for Clinical Research, Osaka National Hospital, Osaka, ${ }^{8}$ Department of Cardiology, Saiseikai Yamaguchi Hospital, Yamaguchi, ${ }^{9}$ Department of Cardiology, Hakodate Medical Association Hospital, Hakodate, ${ }^{10}$ Sasagawa Clinic, Niigata, ${ }^{11}$ Medical \& Biological Science, Gunma University, Maebashi and ${ }^{12}$ Department of Internal Medicine, Keio University, Tokyo, Japan

Mailing address: Hiroshi Inoue, MD, The Second Department of Internal Medicine, University of Toyama, 2630 Sugitani, Toyama 930-0194, Japan. E-mail: hiroshi@med.u-toyama.ac.jp

ISSN-1346-9843 doi:10.1253/circj.CJ-09-0802

All rights are reserved to the Japanese Circulation Society. For permissions, please e-mail: cj@j-circ.or.jp 


\begin{tabular}{lccc|}
\hline \multicolumn{4}{c}{ Table 1. Clinical Characteristics of Japanese Patients With AF } \\
& $\begin{array}{c}\text { Men } \\
(\mathbf{n = 2 , 0 2 8})\end{array}$ & $\begin{array}{c}\text { Women } \\
(\mathbf{n}=\mathbf{8 6 4})\end{array}$ & P value \\
Age (years) & $69.4 \pm 9.4$ & $72.6 \pm 8.5$ & $<0.001$ \\
$\quad \geq 75$ years (\%) & 32.0 & 44.5 & $<0.001$ \\
Chronic AF* (\%) & 68.8 & 66.1 & 0.199 \\
& $(1,062 / 1,543)$ & $(462 / 699)$ & \\
BMI (kg/m $\left.{ }^{2}\right)$ & $23.8 \pm 3.2$ & $23.4 \pm 4.1$ & $<0.001$ \\
CHF (\%) & 17.0 & 27.1 & $<0.001$ \\
Hypertension (\%) & 57.2 & 61.1 & 0.051 \\
DM (\%) & 19.1 & 16.7 & 0.125 \\
Ischemic stroke (\%) & 29.4 & 26.3 & 0.089 \\
VHD (\%) & 10.1 & 21.1 & $<0.001$ \\
MI (\%) & 7.6 & 5.9 & 0.096 \\
HC (\%) & 25.1 & 35.5 & $<0.001$ \\
Drinker (\%) & 46.3 & 5.2 & $<0.001$ \\
Smoker (\%) & 21.2 & 4.3 & $<0.001$ \\
CHADS2 score & $1.88 \pm 1.33$ & $2.05 \pm 1.29$ & $<0.001$ \\
\hline
\end{tabular}

Data are mean \pm SD or $\%$ of patients.

*In the myocardial infarction and stroke categories; subtypes of AF were not specifically determined.

$\mathrm{AF}$, atrial fibrillation; $\mathrm{BMI}$, body mass index; $\mathrm{CHF}$, congestive heart failure; DM, diabetes mellitus; MI, myocardial infarction; VHD, valvular heart diseases including valve replacement; $\mathrm{HC}$, hypercholesterolemia.

prior stroke, myocardial infarction (MI) or AF began in January 2005 and ceased in December 2006.

\section{Study Population}

Patients aged 20-90 years were eligible for enrollment if they had at least 1 of the 3 cardiovascular diseases (stroke, MI or AF). The study protocol was approved by an Institutional Review Board at each participating site and all patients gave informed consent. Those in the AF category, and those in the stroke and MI categories who also had AF, comprised the study subjects for this subanalysis of J-TRACE. Those in the recovery phase of acute $\mathrm{MI}$ or acute stroke were not eligible for enrollment in J-TRACE.

\section{Baseline Characteristics}

All subtypes of AF were included. AF was diagnosed electrocardiographically using standard diagnostic criteria. Risk factors and comorbidities were collected from the medical record as baseline data. Among them were hypertension, diabetes mellitus, hypercholesterolemia, valvular diseases, MI, ischemic stroke, congestive heart failure, smoking, and drinking. Regular use of medications, including anticoagulants, antiplatelet agents, and drugs for hypercholesterolemia, hypertension, and diabetes mellitus, was also determined from the medical record. Each patient's CHADS2 score $^{15}$ was calculated to determine the level of cardioembolic risk: 1 point was given for advanced age ( $\geq 75$ years), hypertension, congestive heart failure, or diabetes mellitus, and 2 points for prior stroke or transient ischemic attack.

\section{Statistical Analysis}

Continuous variables are shown as the mean \pm SD, and categorical variables as percentages. Continuous variables were compared by analysis of variance or Student's t-test, and categorical variables with the chi-square test, with $\mathrm{P}<0.05$ considered significant.

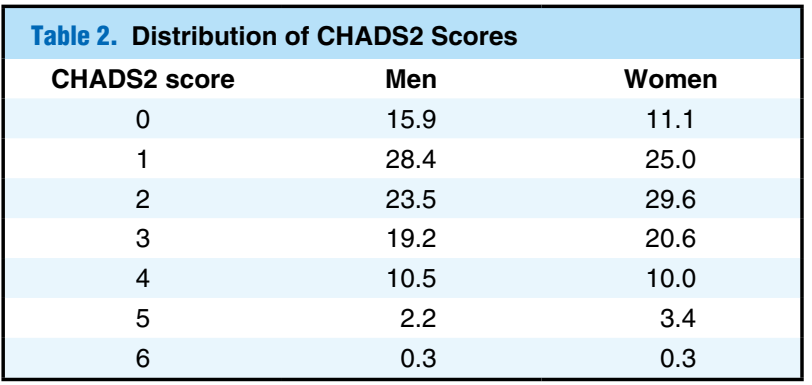

Figures are \% of patients.

$\mathrm{P}<0.001$ between men and women.

\begin{tabular}{|ccccc|}
\hline \multirow{6}{*}{ Table 3. Age and CHADS2 Score } & \\
& \multicolumn{4}{c|}{ Age } \\
\cline { 2 - 4 } & $<65$ years & $\mathbf{6 5 - 7 4}$ years & $\geq 75$ years & \\
Men & $1.24 \pm 1.12$ & $1.63 \pm 1.22$ & $2.74 \pm 1.17$ & \\
& $(\mathrm{n}=572)$ & $(\mathrm{n}=808)$ & $(\mathrm{n}=648)$ & $<0.001$ \\
Women & $1.38 \pm 1.16$ & $1.57 \pm 1.14$ & $2.72 \pm 1.12$ & \\
& $(\mathrm{n}=153)$ & $(\mathrm{n}=326)$ & $(\mathrm{n}=385)$ & $<0.001$ \\
\hline
\end{tabular}

Data are mean \pm SD.

\section{Results}

\section{Risk Factor Profile}

A total of 2,892 patients (2,028 men, 864 women; mean age, 70.3 years) with AF comprised the study group. Numbers of patients and their mean age in the 3 categories were as follows: AF category, 1,543 men (68.9 \pm 9.6 years old) and 699 women (72.4 \pm 8.5$)$; stroke category, 399 men (70.6 \pm 8.4$)$ and 141 women (73.0 \pm 8.3$)$; MI category, 86 men (71.7 \pm 8.1$)$ and 24 women (75.3 \pm 8.0$)$. Their clinical characteristics are summarized in Table 1. Some of the characteristics exhibited differences by sex. Women were older $(\mathrm{P}<0.001)$, and more frequently had congestive heart failure $(\mathrm{P}<0.001)$, hypertension $(\mathrm{P}=0.051)$, valvular diseases or valve replacement $(\mathrm{P}<0.001)$, and hypercholesterolemia $(\mathrm{P}<0.001)$ than the men, but drank $(\mathrm{P}<0.001)$ and smoked $(\mathrm{P}<0.001)$ less frequently than men. The proportion of subjects aged 75 years or older was higher and body mass index was slightly but significantly lower in women than in men $(\mathrm{P}<0.001$, each case). The prevalences of chronic AF, diabetes mellitus, MI, and ischemic stroke did not differ between men and women.

The CHADS2 score was slightly but significantly higher in women than in men (Table $1, \mathrm{P}<0.001$ ) because of their higher prevalence of older age ( $\geq 75$ years), hypertension, and congestive heart failure. The distribution of CHADS2 scores differed significantly between men and women (Table 2, $\mathrm{P}<0.001)$. It increased with age for both men and women, but did not differ between men and women in any age group (Table 3).

\section{Medications}

Medications are summarized in Table 4. Use of warfarin and antiplatelet agents did not differ between men and women. Reflecting the differences in prevalence of hypertension and hypercholesterolemia between men and women, drugs for the treatment of these diseases were used more frequently in women than in men $(\mathrm{P}<0.001$, each case $)$. In contrast, use of antidiabetic drugs was similar in men and women.

There were no apparent sex-related differences in the rate of use of warfarin or aspirin at any CHADS2 score (Table 5). 


\begin{tabular}{|c|c|c|c|}
\hline & Men & Women & $P$ value \\
\hline Warfarin & 73.1 & 72.7 & 0.807 \\
\hline Antiplatelet agents & 37.9 & 36.0 & 0.328 \\
\hline Aspirin & 32.1 & 30.8 & 0.504 \\
\hline Ticlopidine & 5.0 & 5.0 & 0.316 \\
\hline Cilostazol & 2.0 & 1.3 & 0.191 \\
\hline Antihypertensives & 71.8 & 78.8 & $<0.001$ \\
\hline ACEI & 17.4 & 14.8 & 0.087 \\
\hline ARB & 28.4 & 32.2 & 0.039 \\
\hline$\beta$-blockers & 21.4 & 21.3 & 0.927 \\
\hline Calcium antagonists & 36.4 & 42.5 & 0.002 \\
\hline Diuretics & 18.6 & 33.4 & $<0.001$ \\
\hline Lipid-lowering drugs & 16.7 & 26.4 & $<0.001$ \\
\hline Statins & 14.9 & 23.7 & $<0.001$ \\
\hline Antidiabetic drugs & 10.6 & 10.9 & 0.825 \\
\hline Oral & 8.7 & 8.6 & 0.921 \\
\hline Insulin & 1.4 & 2.2 & 0.111 \\
\hline
\end{tabular}

Data are \% of patients.

Only major drugs for treatment of comorbidities and prevention of thromboembolism are listed (see Uchiyama et al ${ }^{18}$ for more detailed information on medications in J-TRACE).

$\mathrm{ACEl}$, angiotensin converting enzyme inhibitors; ARB, angiotensin II receptor blockers.

Warfarin usage differed significantly among CHADS2 scores in both men $(\mathrm{P}<0.001)$ and women $(\mathrm{P}=0.001)$. It increased gradually from approximately $60 \%$ to $80 \%$ as the score increased from 0 to 3 for both men and women; thereafter it reached a plateau, except in the case of women with a score of 6. Aspirin usage also differed significantly among CHADS2 scores in men $(\mathrm{P}=0.008)$, but not in women $(\mathrm{P}=0.852)$. It did not show any apparent score-dependent increase as observed in the case of warfarin usage.

\section{Discussion}

The major findings of the present study are as follows. First, there were sex-related differences in the risk factor profile and medications of patients with AF recruited in J-TRACE. Women were older and more frequently had hypertension, valvular diseases, congestive heart failure, and hypercholesterolemia than men. The prevalence of diabetes mellitus, ischemic stroke, and MI did not differ between men and women. Second, CHADS2 score was consequently slightly but significantly higher in women than in men with AF. This sex-related difference could be largely related to the higher proportion of women aged 75 years or older. Third, no sexrelated differences in the use of warfarin or aspirin were observed at any CHADS2 score.

\section{Risk Factor Profile of Patients With AF}

Reports from Western countries ${ }^{6-10}$ suggest that sex-related differences could exist in the risk factors for cardiovascular diseases of patients with AF. In the present study, mean age was higher and the prevalence of hypertension also tended to be higher in women than in men, consistent with the previous reports $;{ }^{6-9}$ however, the prevalence of congestive heart failure was also higher in women than in men in the present study, a finding that is inconsistent with those reports from Western countries. ${ }^{6-9}$ Notably, the prevalence of diabetes mellitus and of a prior history of ischemic stroke were not

\begin{tabular}{|cccccc|}
\hline \multirow{2}{*}{$\begin{array}{c}\text { Table 5. Use of Warfarin and Aspirin at Each CHADS2 Score } \\
\text { CHADS2 } \\
\text { score }\end{array}$} & \multicolumn{2}{c|}{ Warfarin use (\%) } & & \multicolumn{2}{c|}{ Aspirin use (\%) } \\
\cline { 2 - 3 } \cline { 5 - 6 } 0 & 57.9 & 61.4 & & 32.3 & 28.1 \\
1 & 68.9 & 66.2 & & 33.9 & 31.4 \\
2 & 74.8 & 73.8 & & 30.6 & 32.0 \\
3 & 84.9 & 77.0 & & 26.9 & 30.3 \\
4 & 81.1 & 87.2 & & 34.4 & 29.1 \\
5 & 77.3 & 79.3 & & 31.3 & 27.6 \\
6 & 83.3 & 66.7 & & 83.3 & 66.7 \\
$P$ value & $<0.001$ & 0.001 & & 0.008 & 0.852 \\
\hline
\end{tabular}

consistent. ${ }^{6-9}$

Cohort studies of the general population in Japan have indicated that the prevalences of hypertension and diabetes mellitus are higher in men than in women. ${ }^{19-21}$ The prevalence of cardiac diseases was not higher in women than in men with $\mathrm{AF}^{4,5}$ so the higher prevalences of hypertension and congestive heart failure in women with $\mathrm{AF}$ found in the present study do not simply reflect the prevalence of these diseases in the general population of Japan. Valvular disease is a well-known risk factor for AF, ${ }^{22}$ especially for Japanese women. ${ }^{23}$ Drinking and smoking could promote the development of $\mathrm{AF}^{22-25}$ and were present more frequently in men than in women in the present study, as in the general population of Japan. ${ }^{4,5,19-21}$ The electrophysiological properties of the atria differ between men and women, ${ }^{26}$ so greater comorbidity and age might be required for AF to develop in women than in men.

\section{Thromboembolic Risk}

A sex difference in CHADS2 score was found in the present study, a finding consistent with the ATRIA study. ${ }^{7}$ In the Euro Heart Survey the score might have been higher in women than in men, because mean age and the prevalences of hypertension, diabetes mellitus, and prior ischemic stroke were significantly higher in women than in men. ${ }^{9}$ In some studies the levels of biomarkers of a prothrombotic state were higher in women with $\mathrm{AF}$ than in men with $\mathrm{AF}{ }^{27,28}$ These findings could explain the inclusion of female sex as a risk factor in some schemes for predicting thromboembolic events in patients with AF. ${ }^{13,14}$ In fact, among patients with acute stroke, embolic infarction is observed more frequently in women than in men. ${ }^{29}$ It is difficult to determine the reasons for the sex-related difference in thromboembolic risk; however, some components of the CHADS2 score were observed more frequently in women in the ATRIA study, ${ }^{7}$ Euro Heart Survey, ${ }^{9}$ and in the present study.

\section{Medications}

Registry studies in Western countries have indicated that warfarin usage does not differ between men and women., In the present study, the rate of warfarin usage did not differ between men and women as a whole nor did it differ between them at any CAHDS2 score (Table 5). Warfarin usage is at present not necessarily less frequent in women than in men, as reported in earlier registry ${ }^{6}$ and community-based cohort ${ }^{30}$ studies.

Use of aspirin and antidiabetic drugs was similar in men and women; however, drugs for hypertension and hypercholesterolemia were used more frequently by women than by men. The latter finding might reflect the sex-related differ- 
ences in the prevalence of these diseases in the present study.

\section{Study Limitations}

First, enrollment of consecutive patients with stroke, MI, and $\mathrm{AF}$ was recommended, but may not necessarily have occurred at each participating site and this possible selection bias could have affected the present results. Second, data for subjects with AF were collected from 3 categories of J-TRACE, ${ }^{17,18}$ possibly resulting in increased prevalences of ischemic stroke and MI. However, this might not necessarily have affected sex-related differences in the frequency of these diseases in the present study. Actually, when only patients of AF category were analyzed, the results did not differ in terms of sex-related differences in mean age, CHADS2 score, and prevalences of heart failure, hypertension, smoking, drinking habit and warfarin usage (data not shown). Third, the study design of the J-TRACE did not define the diagnostic criteria of comorbidities, including hypertension, hypercholesterolemia and others; however, data of comorbidities were collected from the medical record. If strict diagnostic criteria of comorbidities were used, the present results would not have changed greatly. Finally, the intensity of anticoagulation was not determined systematically, and follow-up data are not yet available.

\section{Clinical Implications}

Our findings indicate sex-related differences in the clinical risk factor profile of patients with $\mathrm{AF}$, with the CHADS2 score slightly but significantly higher in women with AF than in men with AF in the clinical setting in Japan. Further follow-up studies are required to elucidate the effects of these sex-related differences on subsequent thromboembolic events.

\section{Acknowledgment}

This study was supported by the Japan Heart Foundation, Tokyo, Japan.

\section{Disclosure}

There is no conflict of interest to declare.

\section{References}

1. Feinberg WM, Blackshear JL, Laupacis A, Kronmal R, Hart RG. Prevalence, age distribution, and gender of patients with atrial fibrillation. Arch Intern Med 1995; 155: 469-473.

2. Go AS, Hylek EM, Phillips KA, Chang YC, Henoult LE, Selby JV, et al. Prevalence of diagnosed atrial fibrillation in adults: National implications for rhythm management and stroke prevention: The AnTicoagulation and Risk factors In Atrial fibrillation (ATRIA) study. JAMA 2001; 285: 2370-2375.

3. Majeed A, Moser K, Carroll K. Trends in the prevalence and management of atrial fibrillation in general practice in England and Wales, 1994-1998: Analysis of data from the general practice research database. Heart 2001; 86: 284-288.

4. Inoue H, Fujiki A, Origasa H, Ogawa S, Okumura K, Kubota I, et al. Prevalence of atrial fibrillation in the general population of Japan: An analysis based on periodic health examination. Int J Cardiol 2009; 137: 102- 107 .

5. Iguchi Y, Kimura K, Aoki J, Kobayashi K, Terasawa Y, Sakai K, et al. Prevalence of atrial fibrillation in community-dwelling Japanese aged 40 years or older in Japan: Analysis of 41,436 non-employee residents in Kurashiki-City. Circ J 2008; 72: 909-913.

6. Humphries KH, Kerr CR, Connolly SJ, Klein G, Boone JA, Green $\mathrm{M}$, et al. New-onset atrial fibrillation: Sex differences in presentation, treatment, and outcome. Circulation 2001; 103: 2365-2370.

7. Fang MC, Singer DE, Chang Y, Hylek EM, Henault LE, Jensvold $\mathrm{NG}$, et al. Gender differences in the risk of ischemic stroke and peripheral embolism in atrial fibrillation: The AnTicoagulation and Risk factors In Atrial fibrillation (ATRIA) study. Circulation 2005; 112: $1687-1691$.
8. Gomberg-Maitland M, Wenger NK, Feyzi J, Lengyel M, Volgman AS, Petersen P, et al. Anticoagulation in women with non-valvular atrial fibrillation in the stroke prevention using an oral thrombin inhibitor (SPORTIF) trials. Eur Heart J 2006; 27: 1947-1953.

9. Dagres N, Nieuwlaat R, Vardas PE, Andersen D, Levy S, Cobbe S, et al. Gender-related differences in presentation, treatment, and outcome of patients with atrial fibrillation in Europe: A report from the Euro Heart Survey on atrial fibrillation. J Am Coll Cardiol 2007; 49: 572-577.

10. Kerr CR, Humphries K. Gender-related differences in atrial fibrillation. J Am Coll Cardiol 2005; 46: 1307-1308.

11. Friberg J, Scharling H, Gadsboll N, Truelsen T, Jensen GB. Comparison of the impact of atrial fibrillation on the risk of stroke and cardiovascular death in women versus men (the Copenhagen City Heart Study). Am J Cardiol 2004; 94: 889-894.

12. Stewart S, Hart CL, Hole DJ, McMurray JJV. A population-based study of long-term risks associated with atrial fibrillation: 20-year follow-up of the Renfrew/Paisley study. Am J Med 2002; 113: $359-364$.

13. Hart R, Pearce L, McBride R, Rothbart R, Asinger R. Factors associated with ischemic stroke during aspirin therapy in atrial fibrillation: Analysis of 2012 participants in the SPAF I-III clinical trials. Stroke 1999; 30: $1223-1229$.

14. Wang TJ, Massaro JM, Levy D, Vasa RS, Wolf PA, D’Agostino $\mathrm{RB}$, et al. A risk score for predicting stroke or death in individuals with new-onset atrial fibrillation in the community: The Framingham Heart Study. JAMA 2003; 290: 1049-1056.

15. Gage BF, Waterman AD, Shannon W, Boechler M, Rich MW, Radford MJ. Validation of clinical classification schemes for predicting stroke: Results from the National Register of Atrial Fibrillation. JAMA 2001; 285: 2864-2870.

16. Atrial Fibrillation Investigators. Risk factors for stroke and efficacy of antithrombotic therapy in atrial fibrillation: Analysis of pooled data from five randomized controlled trials. Arch Intern Med 1994; 154: $1449-1457$.

17. Origasa $\mathrm{H}$, Goto $\mathrm{S}$, Uchiyama S, Shimada K, Ikeda $\mathrm{Y}$, on behalf of the J-TRACE Investigators. The Japan Thrombosis Registry for Atrial Fibrillation, Coronary or Cerebrovascular Events (J-TRACE): A nation-wide, prospective large cohort study; the study design. Circ J 2008; 72: 991-997.

18. Uchiyama S, Shibata Y, Hirabayashi T, Mihara B, Hamashige N, Kitagawa K, et al for the J-TRACE Investigators. Risk factor profiles in patients with stroke, myocardial infarction, and atrial fibrillation: The Japan Thrombosis Registry for Atrial Fibrillation, Coronary or Cerebrovascular Events (J-TRACE). J Stroke Cerebrovasc Dis (in press).

19. Ohsawa M, Okayama A, Okamura T, Itai K, Nakamura M, Tanno $\mathrm{K}$, et al for the NIPPON DATA80 Research Group. Mortality risk attributable to atrial fibrillation in middle-aged and elderly people in the Japanese general population: Nineteen-year follow-up in NIPPON DATA80. Circ J 2007; 71: 814-819.

20. Kubo M, Kiyohara Y, Kato I, Tanizaki Y, Arima H, Tanaka K, et al. Trends in the incidence, mortality, and survival rate of cardiovascular disease in a Japanese community: The Hisayama Study. Stroke 2003; 34: 2349-2354.

21. Iso H, Sato S, Kitamura A, Imano H, Kiyama M, Yamagishi K, et al. Metabolic syndrome and the risk of ischemic heart disease and stroke among Japanese men and women. Stroke 2007; 38: 17441751.

22. Benjamin EJ, Levy D, Vaziri SM, D'Agostino RB, Belanger AJ, Wolf PA. Independent risk factors for atrial fibrillation in a population-based cohort: The Framingham Heart Study. JAMA 1994; 271: $840-844$.

23. Fujishima M. Cardiac diseases as risk for cerebrovascular diseases. Junkanki Senmon-i 1998; 6: 19-26 (in Japanese).

24. Djousse L, Levy D, Benjamin EJ, Blease S, Russ A, Larson MG, et al. Long-term alcohol consumption and the risk of atrial fibrillation in the Framingham study. Am J Cardiol 2004; 93: 710-713.

25. Heerings J, Kors JA, Hofman A, van Rooij FJA, Witteman JCM. Cigarette smoking and risk of atrial fibrillation: The Rotterdam Study. Am Heart J 2008; 156: 1163-1169.

26. Tse HF, Oral H, Pelosi F, Knight BP, Strickberger SA, Morady F. Effect of gender on atrial electrophysiologic changes induced by rapid atrial pacing and elevation of atrial pressure. J Cardiovasc Electrophysiol 2001; 12: 986-989.

27. Conway DS, Heeringa J, Van der Kuip DA, Chin BS, Hofman A, Witteman JC, et al. Atrial fibrillation and the prothrombotic state in the elderly: The Rotterdam study. Stroke 2003; 34: 413-417.

28. Feinberg WM, Pearce LA, Hart RG, Cushman M, Cornell ES, Lip GY, et al. Markers of thrombin and platelet activity in patients with 
atrial fibrillation: Correlation with stroke among 1531 participants in the Stroke Prevention in Atrial Fibrillation III study. Stroke 1999; 30: $2547-2553$.

29. Appelros P, Stegmayr B, Terent A. Sex differences in stroke epidemiology: A systematic review. Stroke 2009; 40: 1082-1090.

30. Sudlow M, Thomson R, Thwaites B, Rodgers H, Kenny RA. Prevalence of atrial fibrillation and eligibility for anticoagulants in the community. Lancet 1998; 352: 1167-1171.

\section{Appendix 1}

\section{J-TRACE Steering Committee}

Yasuo Ikeda, Keio University School of Medicine (Chair); Kazuyuki Shimada, Jichi Medical School; Shinichiro Uchiyama, Tokyo Women's Medical University; Shinya Goto, Tokai University School of Medicine; and Hideki Origasa, University of Toyama School of Medicine and Pharmaceutical Sciences

Secretariat

Hiroko Usami, BIOMEDIS INTERNATIONAL LTD

Regional Coordinators

Hokkaido Kiyohiro Houkin, Jyoji Nakagawara, Kazuaki Shimamoto, Hiroyuki Tsutsui

Tohoku Kunio Shirato, Hiroaki Shimokawa, Akifumi Suzuki, Yasuo Terayama

Kanetsu Yoshifusa Aizawa, Akira Imai, Masahiko Kurabayashi, Ban Mihara, Yasuo Sasagawa

Chiba/Saitama Nobuo Araki, Toshio Fukutake, Issei Komuro, Fumitaka Ohsuzu

Tokyo/Kanagawa Masahiko Aosaki, Kazuo Kimura, Norihiro Suzuki, Makoto Takagi, Shigeharu Takagi, Teruo Takano, Teruhisa Tanabe

Tokai Haruo Hirayama, Toyoaki Murohara, Takeshi Nakano, Gen Sobue

Kansai Masatsugu Hori, Yukihiro Koretsune

Hokuriku Shunro Endo, Hiroshi Inoue, Kouji Kajinami

Chugoku/Shikoku Naohisa Hamashige, Shotai Kobayash, Masayasu Matsumoto, Masunori Matsuzaki

Kyushu Yoichiro Hashimoto, Hisao Ogawa, Yasushi Okada, Shuuichi Okamatsu

\section{Participating Investigators}

Hokkaido Takeo Abumiya, Minoru Ajiki, Takeo Baba, Tomoo Furumoto, Shuuzaburou Fukuyama, Masatada Fukuoka, Takayuki Hirabayashi, Tooru Hasegawa, Kiyohiro Houkin, Katsuhisa Ishii, Satoshi Koyama, Kenji Kamiyama, Tetsuro Kohya, Satoshi Kuroda, Tsukasa Kubota, Takaaki Kato, Michifumi Kyuma, Takeo Murahashi, Tomoaki Matsumoto, Jyoji Nakagawara, Daigo Nagahara, Hiroshi Oimatsu, Toshiaki Osato, Hitoshi Ooiwa, Kazuhiro Sako, Motoi Sasaki, Toshihiro Shimizu, Yoshinobu Seo, Tetsuro Shoji, Tsukasa Satoh, Yasukuni Shikano, Mitsunori Shimazaki, Makoto Senoo, Yoshitoki Takagawa, Akihito Tsuchida, Shiho Takagawa, Hideki Takizawa, Shigemichi Tanaka, Hidekazu Takada, Shuuji Yonekura

Tohoku Morio Aihara, Mituaki Hatanaka, Yasuhiro Ishibashi, Hiroyuki Kuki, Takahiko Kikuchi, Yuichi Nozaki, Ayumu Ohnuma, Yukio Onodera, Hiroyuki Oosawa, Satoko Obara, Akifumi Suzuki, Nobuyuki Shiba, Hiroaki Takahashi, Kenichi Tamura, Hajime Yasuda

Kanetsu Kazunori Akaji, Takenori Akiyama, Tsuneo Fujita, Hiroshi Furushima, Mikio Fujimoto, Shintaro Gomi, Kenji Hiraga, Satoru Hirono, Masahiro Hirose, Akira Imai, Masahiro Inoue, Toshiya Iwasaki, Kunihiko Imai, Masaki Jinbo, Yoshiaki Kaneko, Kiminori Kato, Hiroshi Kamiyama, Bun-ichi Kato, Ban Mihara, Masatsugu Morikawa, Mitsunobu Murata, Tatsuru Mihara, Takahide Nagashima, Shibata/Kitakanbara J-TRACE team, Toyoshi Sasaki, Yasuo Sasagawa, Kaoru Suzuki, Keisuke Suzuki, Hiroshi Shimizu, Norisuke Satori, Yutaka Tomita, Yoshio Tanizaki, Toshinori Takahashi, Yasuhiko Yamauchi

Chiba/Saitama Nobuo Araki, Toshio Fukutake, Kouichi Honma, Yoshihiro Iijima, Shoichi Ito, Masatoshi Kusuhara, Yoichi Kuwabara, Yoshio Kobayashi, Kazutaka Matsui, Shinji Matsuda, Takashi Nakazato, Kyoichi Nomura, Toshio Nagai, Fumitaka Ohsuzu, Hideki Shige, Shigeki Tanaka, Kazuo Yamakawa
Tokyo/Kanagawa Hisanao Akiyama, Kuniya Asai, Eiiti Akiyama, Toshiaki Ebina, Mitsuaki Endo, Tsutomu Endo, Eri Furukawa, Hirotaka Fujita, Kazuki Fukui, Shinya Fukazawa, Taiji Furukawa, Shinya Goto, Yoshinari Goseki, Haruhiko Hoshino, Kazuhiro Hara, Kei Hatori, Jynya Hosoda, Satoshi Hida, Masaharu Hirano, Kazunori Iwade, Noriaki Iwahashi, Aritomo Inoue, Shinobu Imai, Taizo Ishiyama, Kensuke Ishii, Sugao Ishiwata, Yuji Ikari, Kohei Iguchi, Yutaka Kitamura, Eitaro Kodani, Yasuhisa Kitagawa, Haruhisa Kato, Hirotaka Kato, Takashi Kiyanagi, Ikuyoshi Kusama, Kazuo Kimura, Noriyuki Kawaura, Tsukasa Koboyashi, Takahide Kohro, Kimio Kikushima, Atsumi Kume, Mitsuharu Kawamura, Kazuhiro Muramatsu, Kohei Matsushita, Yoko Morita, Yoshino Minoura, Jun Masuda, Yukiko Morita, Takayuki Mitsuhashi, Nobuhiko Maejima, Minako Murayama, Takamichi Miyamoto, Kagari Matsudaira, Manabu Miyagi, Hiroyoshi Morino, Riichiro Nakayama, Takehiko Nagao, Hiroshi Nishimura, Masashi Nakamaru, Tatsuya Nakachi, Tomoyori Nakatogawa, Takeshi Nakagawa, Masyayuki Nakao, Akihiro Niwa, Hirotoshi Ohmura, Hiroyuki Ozaki, Syuuji Ono, Jun Okuda, Yasuo Ohkusu, Yuji Otsuka, Hisako Omori, Miei Shimura, Tomoaki Shimizu, Takahiro Shibata, Ryouma Shibue, Fumio Saito, Shigeru Nogawa, Kenichiro Saka, Toshihiko Saito, Toshiak Sato, Yutaka Shiina, Naohisa Shindo, Shigeharu Takagi, Makoto Takagi, Kengo Tsukahara, Tatuya Takahashi, Itaru Takamisawa, Tetsu Takamizawa, Naoyuki Takahashi, Shigemasa Tani, Koichi Tamura, Shinichi Takahashi, Hidehito Takase, Takeshi Tadera, Hirokazu Tanaka, Teruhisa Tanabe, Nobuhiro Tanaka, Yasuyoshi Takei, Yoko Takiyama, Shinichiro Uchiyama, Jun Umemura, Hiromi Uno/Terashi, Mikio Usui, Ikuyoshi Watanabe, Masayuki Yotsukura, Hideto Yano, Takeshi Yamakawa, Minako Yoshida Tokai Tetsuo Ando, Mikio Hirayama, Takashi Hara, Kazuhiro Hara, Nozomi Hishikawa, Mizuki Ito, Noboru Imai, Tetsuya Kitamura, Sukenari Koyabu, Noriko Kodama, Osamu Kawakami, Takashi Kameyama, Takahisa Kondo, Masaaki Kanashiro, Toyoaki Murohara, Mamoru Nanasato, Mikiya Nakayabu, Hisayoshi Niwa, Masahiro Oya, Osamu Ohno, Makoto Sugiura, Hidetaka Watanabe

Kansai Ken Araki, Hideki Etani, Ryuzo Fukunaga, Shigetaka Furukado, Hisakazu Fuji, Katsuji Hashimoto, Hiroyuki Hashimoto, Kinji Ishikawa, Kazuo Kitagawa, Yukihiro Koretsune, Akio Kohama, Yoshiyuki Kijima, Shinsuke Nanto, Katsunori Nao, Yoshiyuki Nagai, Keiko Nagano, Osaka Police Hospital's Cardiology, Yutaka Okazaki, Masafumi Tagaya, Tsutomu Takahashi

Hokuriku Hironobu Akao, Shunro Endo, Nakaba Fujioka, Akira Fujiki, Tadakazu Hirai, Jun Harada, Hisanari Ishise, Hiroshi Inoue, Bunji Kaku, Michiya Kubo, Masanori Kyoi, Kiyoo Mori, Koichi Mizumaki, Yutaka Nitta, Takashi Nozawa, Makoto Nonomura, Ryoko Sato, Shutaro Takashima, Yoshihiro Takeuchi, Yoshiharu Taguchi, Kortaro Tanaka, Tsukasa Takabatake, Tomio Taguchi

Chugoku/Shikoku Hitoshi Fukuda, Yuhji Furutani, Kaori Fujibe, Takashi Fujii, Naohisa Hamashige, Naohisa Hosomi, Masahiko Harada, Yuji Hisamatsu, Koji Hirashita, Hijiri Ito, Takahiro Iwami, Shoichi Kato, Juri Kitamura, Masateru Kohno, Mitsuhiro Kitani, Hiromi Koide, Masayasu Kimura, Satoshi Kataoka, Kyoko Kobayashi, Toshiro Miura, Shingo Mitaki, Yasuhiro Manabe, Eiichi Nomura, Koichi Noda, Atsushi Nagai, Kiyoshi Nishino, Manabu Nasu, Takanori Namba, Fumiaki Nakao, Shiro Ono, Eiichi Ohnuki, Kazunori Okada, Takayuki Okamura, Tomohiko Ohshita, Hiroaki Sugiura, Kozaburo Seki, Tsuyoshi Torii, Taketo Tanigawa, Hideo Terasawa, Tsuyoshi Ueyama, Masahiro Yamasaki, Kyounori Yasumoto, Toshihiko Yamagata

Kyushu Yutaka Akatsuka, Yoshihiro Fukumoto, Yasuo Fukuda, Takako Fujiki, Yuji Fukutome, Koji Hiyamuta, Rikuzo Hamada, Yasuo Hayashi, Youichi Hokezu, Yoichiro Hashimoto, Yoshifumi Hirata, Tadashi Hamada, Takeshi Ideguchi, Takuroh Imamura, Masatoshi Koga, Junji Kawagoe, Sunao Kojima, Shigehiko Kumate, Ikuo Misumi, Junnko Mashiba, Takashi Matsuura, Hiroshi Nakane, Toshiyasu Ogata, Hirokuni Ohba, Shuichi Okamatsu, Hideki Oka, Yoshisato Shibata, Satoko Saito, Ichiro Shimada, Kazuhito Tsuruta, Kazuhiro Tashima, Yoshihide Taniwaki, Satoshi Terai, Takeshi Yamada, Hitoshi Yasumoto, Akira Yamada, Tohru Yamawaki 